\title{
Management and outcomes in chronic thromboembolic pulmonary hypertension: from expert centers to a nationwide perspective
}

Escribano-Subías, $\mathrm{P}^{1 \#}$; del Pozo, $\mathrm{R}^{2 \#}$; Román-Broto, $\mathrm{A}^{3}$; Domingo Morera $\mathrm{JA}^{4}$; LaraPadrón, $\mathrm{A}^{5}$; Elías Hernández, $\mathrm{T}^{6}$; Molina-Ferragut, L $\mathrm{L}^{7}$; Blanco, $\mathrm{I}^{8}$; Cortina, $\mathrm{J}^{9}$; Barberà, $\mathrm{JA}^{8}$ on behalf of REHAP* investigators.

REHAP*: Registro Español de Hipertensión Arterial Pulmonar (Spanish Registry of Pulmonary Arterial Hypertension)

${ }^{1}$ Pulmonary Hypertension Unit. RIC group. Cardiology Department, Hospital Universitario 12 de Octubre. Madrid, Spain

${ }^{2}$ Pulmonary Hypertension Unit. Pneumonology Department, Hospital Universitario 12 de Octubre. Madrid, Spain.

${ }^{3}$ Department of Pneumonology. Hospital Universitari Vall d’Hebrón. Universitat Autonoma, Barcelona, and Biomedical Research Networking Center on Respiratory Diseases (CIBERES), Spain.

${ }^{4}$ Department of Pneumonology. Hospital Universitario Miguel Servet. Zaragoza, Spain.

${ }^{5}$ Department of Cardiology. Hospital Universitario de Canarias. Santa Cruz de Tenerife, Spain.

${ }^{6}$ Department of Pneumonology. Hospital Universitario Virgen del Rocío. Sevilla, Spain.

${ }^{7}$ Department of Cardiology. Hospital del Mar. Barcelona, Spain. Heart Diseases

Biomedical Research Group, IMIM (Hospital del Mar Medical Research Institute),

Barcelona, Spain.

${ }^{8}$ Department of Pulmonary Medicine, Hospital Clínic-IDIBAPS, University of Barcelona. Barcelona, Spain; and Biomedical Research Networking Center on Respiratory Diseases (CIBERES), Spain.

${ }^{9}$ Pulmonary Hypertension Unit. Cardiac Surgery Department, Hospital Universitario 12 de Octubre. Madrid, Spain

\# These two authors contributed equally to this work

* A full list of REHAP investigators is given in the Appendix

\section{Corresponding author:}

Pilar Escribano-Subias, MD, $\mathrm{PhD}$

Pulmonary Hypertension Unit. Cardiology Department.

Hospital Universitario 12 de Octubre.

Complutense University, Madrid.

Crtra. Andalucia km 5,400. 28041 Madrid. Spain

E-mail: pilar.escribano.subias@ gmail.com 


\section{ABSTRACT}

BACKGROUND: The Spanish "Registry of Pulmonary Arterial Hypertension" (REHAP), started in 2007, includes chronic thromboembolic hypertension (CTEPH) patients. Based on data provided by this registry and retrospective data from patients diagnosed during 2006 ( $\leq 12$ months since the registry was created), clinical management and long-term outcomes of CTEPH patients are analyzed nationwide for the first time in a scenario of a decentralized organization model of CTEPH management. METHODS AND RESULTS: A total of 391 patients (median [Q1:Q3] age $63.7[48.0 ; 73.3]$ years, 58\% females) with CTEPH included during the period January 1, 2006 - December 31, 2013 in the REHAP registry were analyzed. Rate of pulmonary endarterectomy (PEA) was $31.2 \%$, and highly asymmetric among centers: rate was $47.9 \%$ at two centers designated as CTEPH expert centers, while it was $4.6 \%$ in other centers. Among patients not undergoing PEA, $82 \%$ were treated with therapies licensed for pulmonary arterial hypertension (PAH). Five-year survival rate was $86.3 \%$ for PEA patients, and $64.9 \%$ for non-PEA patients. Among non-PEA patients, presenting proximal lesions ( $42 \%$ of non-referred patients) was associated with a 3 -fold increase in mortality. PEA patients achieved significantly better hemodynamic and clinical outcomes at one-year follow-up compared to non-PEA patients. Patients not being referred for PEA assessment were older and had a worse functional capacity. Older age was the most deterrent factor for non-operability. CONCLUSION: Despite the increase in diagnosis and expertise in PEA-specialized centers, an important percentage of patients do not benefit of PEA in a decentralized organization model of CTEPH management. 


\section{INTRODUCTION}

Chronic pulmonary thromboembolic hypertension $(\mathrm{CTEPH})$ is one of the leading causes of severe pulmonary hypertension $(\mathrm{PH})$ and as such, it is associated with significant morbidity and mortality $[1,2]$. Even though recognition of CTEPH has recently increased [3], epidemiological data are scarce $[3,4]$ and there is an urgent need to identify patients.

Pulmonary endarterectomy (PEA) is the treatment of choice for CTEPH patients given its potential to cure the disease [5], with restoration of hemodynamics to normal or nearly normal and improvement in clinical symptoms in most patients [3, 6, 7]. PEA is performed in highly specialized centers, and outcomes are associated with growing expertise in the technique, optimal patient selection, and better perioperative management $[3,8]$. Until the launch in 2015 of riociguat -currently the only treatment licensed for CTEPH-, which has demonstrated to significantly improve exercise capacity and PVR in CTEPH patients deemed inoperable or those with residual PH after PEA $[9,10]$, patients were treated with medical therapies proven to be effective in pulmonary arterial hypertension $(\mathrm{PAH})[5]$.

Current knowledge on the outcomes associated with the management of CTEPH arises precisely from these PEA-specialized centers $[3,11]$. However, this information is far from capturing outcomes of CTEPH patients at a nationwide perspective since countryspecific management issues, and specifically how referral for PEA assessment is undertaken (i.e. mandatory and centralized in reference centers vs. optional and based on a decentralized decision-making model), may greatly influence outcomes at this level. These kinds of analyses, which are fundamental to outline policies for CTEPH 
management, are scarce and only available from countries where a centralized model is followed $[3,12]$.

A national observational registry of pulmonary arterial hypertension (REHAP) has been running in Spain since 2007 providing valuable information about CTEPH epidemiology and survival [4]. This registry is one of the largest currently in place in Europe. One of its objectives was to evaluate the clinical management of CTEPH patients and long-term outcomes under a nationwide perspective, according to the management organization model established. This model is characterized for being decentralized, with management of patients (i.e. diagnosis and treatment, some of them including PEA) taking place at so-designated PH-specialized centers and at general hospitals. In centers not performing PEA, decision regarding referral for PEA assessment is left to doctor's judgment. Two of the PH-specialized centers bring together most CTEPH patients and are designated as expert centers.

The REHAP offers therefore a unique opportunity to analyze from a nationwide perspective how patients are treated and long-term outcomes according to treatment in a decentralized model of CTEPH management. This is also especially interesting since only a few long-term evidence of outcomes in CTEPH patients receiving PAH-specific therapies is currently available [3]. Other data of interest such as how patient selection for PEA assessment is undertaken when left to doctor's judgment is also provided. 


\section{METHODS}

\subsection{Study subjects}

Analyzed CTEPH patients were included in the REHAP registry from January 1, 2006 to December 31, 2013. Patients diagnosed during 2006 ( $\leq 12$ months since the registry was created) were included retrospectively, and prospectively thereafter. Centers reporting data to the registry included $31 \mathrm{PH}$-specialized centers and general hospitals, which covered 15 of the 17 administrative regions of Spain. Two of these centers managing more than $50 \mathrm{CTEPH}$ patients (those belonging to the health administrative area and those being referred for PEA assessment) are considered CTEPH expert centers. As such, they participate at the International CTEPH registry [11].

Study design, inclusion criteria and data collection have been previously described [4, 13]. Summarizing, registered patients were older than 14 years and met the modified definition of CTEPH [14] and pre-specified hemodynamic criteria by right heart catheterization $(\mathrm{RHC})$ before study entry (mean pulmonary artery pressure $[\mathrm{mPAP}] \geq$ $25 \mathrm{~mm} \mathrm{Hg}$, pulmonary capillary wedge pressure or left ventricular end-diastolic pressure $\leq 15 \mathrm{mmHg}$ or $16-18 \mathrm{~mm} \mathrm{Hg}$ if justified [11] and PVR $\geq 3$ Wood units). All patients showed perfusion defects by ventilation/perfusion lung scintigraphy and CT angiography consistent with CTEPH. Patients showing a $\mathrm{FEV}_{1}<60 \%$ were included if they met all other inclusion criteria. All patients received anticoagulation therapy for at least three months before a CTEPH diagnosis, and continued receiving it chronically.

\subsection{Follow-up}

Data were obtained from assessments routinely performed in clinical practice, and included demographic (age and gender) and anthropometric parameters, PH clinical 
characteristics, and diagnostic parameters (echocardiography and RHC). Lesion proximity was assessed by computed tomographic angiography. Lesions affecting main and lobar arteries were considered proximal lesions. Decision to operate was based on the judgment of the multidisciplinary team of each centre and was based on lesion accessibility, hemodynamic status and presence of co-morbidities. Haemodynamic residual $\mathrm{PH}$ was defined as having a $\mathrm{mPAP} \geq 25 \mathrm{~mm} \mathrm{Hg}$ at rest [5]. Clinically relevant PH was defined as also having a PVR $\geq 5$ Wood units [15]. Both, all-cause and PHrelated causes of death (heart failure and sudden death) were collected. The protocol was reviewed and approved by the Institutional Review Board of the Hospital de Cruces, Bilbao, Spain.

\subsection{Statistical analysis}

Continuous variables were expressed as mean (standard deviation [SD]) or as median with first and third quartiles $(\mathrm{Q} 1 ; \mathrm{Q} 3)$ when not normally distributed. Comparisons were made using the paired $t$ test for parametric data or the U-Mann Whitney test for nonparametric data. Categorical variables were expressed as $\mathrm{n}(\%)$ and compared using the Chi-square test or Fisher's exact test, as appropriate. All P-values were two-sided, with a P-value $<0.05$ being considered statistically significant.

Univariate Cox's proportional-hazard regression models were used to assess the relationship between patient characteristics and both, the likelihood to undergo PEA and mortality. The selection of patients' characteristics potentially associated with mortality or with the likelihood of undergoing PEA was made on the basis of published data $[3,6$, $11,16]$ and own expertise. Only variables available in $>70 \%$ patients were chosen. Colinearity between variables was examined. Variables identified in this analysis (P-value 
$<0.2$ ) were included in a forward stepwise multivariate Cox's proportional hazards model in order to identify independent risk factors. A 95\% confidence interval (CI) was considered in PEA and mortality models.

Survival was estimated using the Kaplan-Meier analysis. The study date of entry was defined as the date of the first diagnostic RHC. Patients were followed-up until the censoring date of December 31, 2014 or the date of death. For survival analysis, patients lost to follow-up were censored at the time of their last visit or observation. All-cause mortality was defined as the end-point and the log-rank test was used for comparison between groups. Outcomes at one year were also analyzed (follow-up analysis). For this purpose, the visit date closer to one year was chosen. Patients included in the follow-up analysis were those with a follow-up visit 4 months after PEA or at initiation of medical treatment when no PEA was performed. Overall change (Delta value) was calculated for the most prominent variables indicating prognosis. All statistics were performed using SPSS version 17 (SPSS Inc., Chicago, IL, USA). 


\section{RESULTS}

\subsection{Study population}

A total of 391 CTEPH patients meeting the inclusion criteria had been included in the registry by December 2013, and constituted the study population. Patients presented a median age $(\mathrm{Q} 1 ; \mathrm{Q} 3)$ of $63.7(48.0 ; 73.3)$, were women in $58 \%$ of cases and had a severe clinical and hemodynamic condition (Table 1).

\subsection{Treatment at diagnosis}

Among all patients, 122 (31.2\%) underwent PEA during this period (hereafter PEA patients). Of these, 115 PEAs (94\% of all PEAs) took place among the 240 patients (47.9\%) managed at the two expert centers (see Introduction) (figure 1). Median (Q1;Q3) time from diagnosis to PEA was 135 (46;276) days. Compared to non-PEA patients, patients undergoing PEA were younger and male in a higher proportion and had a better functional capacity and a higher mPAP (Table 1).

Among PEA patients, 53 (43.4\%) had received pre-operative treatment with PAHspecific therapies. Compared to patients not receiving pre-operative treatment, those treated had a higher PVR (median [Q1:Q3] 11.0 [7.3;14.9] vs. 7.9 [5.5;11] Wood units; $\mathrm{P}=0.001$ ) and mPAP (mean [SD] 51.1 [11.3] vs. 45.6 [13.2] $\mathrm{mm} \mathrm{Hg} ; \mathrm{P}=0.018$ ), and a reduced cardiac output (mean $[\mathrm{SD}] 3.9[1.2]$ vs. $4.5[1.0] \mathrm{L} \cdot \mathrm{min}^{-1} ; \mathrm{P}=0.008$ ). No other differences were observed with respect to functional class or the 6-minute walking distance (6MWD) (data not shown). Median (Q1;Q3) time from diagnosis to PEA was $195(101 ; 391)$ days in the pre-treated group and $77(28 ; 182)$ days in the non-pre-treated group $(\mathrm{P}=0.001)$ 
Among the 269 patients not undergoing PEA (hereafter non-PEA patients), $220(81.8 \%)$ received PAH-specific therapy, with endothelin receptor antagonists (ERA) and PDE-5i being the most frequently used (Table 2).

\subsection{Outcomes according to treatment}

Survival

One-, 3-, and 5-year survival rates from diagnosis were $96.6 \%, 90.9 \%$ and $86.3 \%$ for PEA patients, and $92.6 \%, 80.7 \%$ and $64.9 \%$ for non-PEA patients $(\mathrm{P}=0.003$, figure 2$)$. Fourteen patients died in the PEA group during the whole study period (11.5\%) and 71 in the non-PEA group (26.4\%). Sudden death and heart failure accounted for $42.9 \%$ deaths among PEA patients and $47.9 \%$ among non-PEA patients. Perioperative complications accounted for 4 deaths after PEA (3.3\%). Within 1 year after PEA, two more patients died. Seventeen patients were lost to follow-up (9 PEA patients [7.4\%] and 8 non-PEA patients [3.0\%]).

Patient characteristics, procedures and other variables of interest potentially associated with greater mortality in univariate analysis are shown in Table 3. Multivariate analyses showed that PEA was independently associated with a 53\% reduction in the risk of death (hazard ratio $[\mathrm{HR}]=0.470 ; 95 \%$ CI 0.228 to $0.971 ; \mathrm{P}=0.042$ ) in the overall population. A higher cardiac output was also an independent protective factor in all patients, while a higher 6MWD was a mild protective factor in the non-PEA and overall groups. Presenting pericardial effusion was in all cases an independent mortality risk factor. Among non-PEA patients, presenting proximal lesions was associated with a 3fold increase in mortality $(\mathrm{HR}=3.158 ; 95 \%$ CI 1.691 to $5.899 ; \mathrm{P}<0.001)$ (figure 3). 


\section{Residual PH after PEA}

In patients for whom RHC assessment was available within 6 months and 1 year after PEA $(\mathrm{N}=97), 40(41.2 \%)$ presented residual PH and 29 (29.9\%) clinically relevant residual PH. Mean (SD) PVR was 2.59 (1.05) Wood units in the group with a favourable outcome, and 7.60 (3.25) Wood units in the group with clinically relevant residual $\mathrm{PH}$.

\section{Follow-up outcomes}

Median follow-up time at the 1-year visit was 365 (256;420) days in the PEA group, and $372(282 ; 464)$ in the non-PEA group. Data were available for 99 PEA and 237 nonPEA patients. At this visit, PEA patients achieved significantly better hemodynamic and clinical outcomes compared to non-PEA patients (figure 4). These better outcomes were also reflected in the lower need for PAH-specific therapy: 16 out of 90 PEA patients (16.2\%) and 183 out of $237(77.2 \%)$ non-PEA patients were receiving medical treatment at this visit $(\mathrm{P}<0.001)$.

\subsection{Patient selection for referral}

Compared to patients assessed at expert centers, those assessed only at local PHspecialized centers / general hospitals (i.e. not being referred to expert centers) had a better hemodynamic condition but were also significantly older (median age was 70.4 vs. 57.1 years; $\mathrm{P}<0.0001$ ) and had a worse functional capacity (median distance walked 305 vs. $368 \mathrm{~m} ; \mathrm{P}=0.002$ ). They presented proximal lesions in a lower proportion (23\% vs. $49 \%$; $\mathrm{P}<0.001)$ (see Table 1 at the Supplemental Materials section, available at the on line version). 


\subsection{Patient selection for PEA}

On multivariate analysis, presenting proximal lesions was the patient characteristic associated with a greater likelihood for operability $(\mathrm{HR}=9.895 ; 95 \% \mathrm{CI} 4.639$ to 21.106; $\mathrm{P}<0.001$ ), including characteristics identified on univariate analysis (see Table 2 at the Supplemental Materials section) better physical status (6MWD, $20 \mathrm{~m}$ ) had only a small effect $(\mathrm{HR}=1.063 ; 95 \% \mathrm{CI} 1.008$ to $1.120 ; \mathrm{P}=0.024)$. Older age was the only independent risk factor for non-operability $(\mathrm{HR}=0.596 ; 95 \% \mathrm{CI} 0.468$ to $0.758 ; \mathrm{P}<$ 0.001) (see fig. 1 at the Supplemental Materials section, available at the on line version).

\section{DISCUSSION}

Our study, based on data provided by the REHAP registry, provides novel useful information regarding diagnosis and outcomes associated to CTEPH patient management from a nationwide perspective vs. PEA-specialized enter in a specific scenario of decentralized decision-making model of referral for PEA assessment. Analysis of the data has revealed the existence of an important gap between operability rate at PEA-specialized centers and that of the country, when considered as a whole. This implies that, despite increasing surgical expertise in these highly specialized centers $[17,18]$ and the better outcomes achieved by patients undergoing PEA as reported here, a decentralized model of CTEPH management may severely impact CTEPH patient prognosis.

Main aspect arising from our analysis is that despite global rate of PEA scale was $31.2 \%$, results were highly asymmetric across the different centers performing PEA. 
Noteworthy is the fact that nearly $95 \%$ of surgeries were performed in the two expert centers, which showed a rate of $47.2 \%$ of PEA among CTEPH patients being assessed in those centers (patients belonging to the health administrative area and those being referred for PEA assessment). On the other hand, PEA rate among the 151 patients not being managed in or referred to these centers $(38.6 \%$ of patients included in this registry) was $4.6 \%$ (7 out of 151). This is important since PEA rate at the two expert centers resembled that of other expert centers (i.e. those participating at the International prospective registry [11]), although comparisons are difficult since these latter figures are based on "operable" patients being assessed by expert teams, and not on "operated" patients as it is our case. Nevertheless, our results show how a wider perspective may better reflect the real scenario in CTEPH patient management compared to that showed by expert centers. This is especially true in decentralized decision-making models, as for instance the national PEA rate in UK $(\sim 50 \%)$ is that of the single PEA center where all patients are mandatorily referred [3], which supports the need of PEA assessment being mandatory for all CTEPH patients, as recommended by guidelines and expert committees [5, 19].

Our study also provides further evidence regarding the better outcomes associated with PEA with respect to survival and hemodynamic condition, compared to those achieved in patients not undergoing this procedure. Most patients not undergoing PEA (82\%) were treated with $\mathrm{PAH}$-specific therapies, despite the fact that therapies available at the time of study had no proven benefit in prospective randomized studies [20-22] and that only highly selected patients deemed inoperable are thought to benefit from this therapy [19]. Hence, our study also provides evidence of long-term outcomes of CTEPH patients receiving one or another treatment thus reflecting again the real situation in 
CTEPH management. However, survival, clinical and hemodynamic outcomes in nonPEA patients -and therefore receiving medical treatment-, have to be interpreted taking into account that not all non-PEA patients were deemed inoperable due to lack of referral in some cases, and the specific characteristics of these patients as a result of the "non-referral selection" in potentially PEA-patients as discussed below. Five-year survival rate was $86.3 \%$ in PEA patients and $64.9 \%$ in non-PEA ones. These survival rates among PEA patients are similar to those reported in other studies [8]. Greater clinical and hemodynamic improvement among PEA patients translated into a low need for medical treatment at the first follow-up visit (15\%). It should be noted that half of PEA patients also received this therapy before the procedure despite the ongoing controversy regarding its benefit $[19,23]$. These patients had in fact a worse hemodynamic status at diagnosis. This practice was associated with a 2.5 -fold increase delay in undergoing PEA $(\mathrm{P}=0.001)$, as also reported in other studies $[6,19]$.

Presenting pericardial effusion, a known independent predictor of mortality in patients with PAH $[24,25]$, was associated with increased mortality both in PEA and non-PEA patients, with a higher effect in the former. In our set of patients prevalence was $9.9 \%$, similar to that reported by Lang et al. [16] in other CTEPH series (14.1\%). Higher cardiac output was inversely related to mortality in both groups of patients, as previously described among CTEPH patients [26]. The protective effect of 6MWD was mild, and evident only in non-PEA patients.

Our study has showed that older patients or those with a worst clinical condition (as assessed by the 6MWT) were less likely to be referred for PEA assessment. This is important since $23 \%$ of patients being managed at local hospitals/ $\mathrm{PH}$-specialized 
centers presented proximal lesions. They were not referred even when their median age was $70.4(58.3 ; 76.4)$ years and they could have benefited from PEA. Moreover, nonPEA patients presenting proximal lesions showed a 3-fold increase in mortality, thus reinforcing the need of making available the benefit of PEA to these patients. Mortality was higher among patients not being treated in expert centers, although this might be due to the fact that patients at expert centers underwent PEA in a much higher proportion.

As expected, presence of a proximal lesion was the strongest patient characteristic leading to operability. Conversely, older age was identified as the main deterrent factor, probably reflecting the low referral rate of relatively old patients. Other potential deterrent factors, these including a worst hemodynamic status or frailty, did not merge as independent factors for operability on multivariate analysis. Although some patients might have refused to undergo PEA, this reason was not collected in the registry.

Some of the limitations to be considered when interpreting these data have been previously discussed. Globally, the voluntary nature of this registry may lead to missing or inconclusive data for analysis, and may also affect epidemiology data. Limitations due to the retrospective inclusion of patients affect only patients included during 2006 (one out of the eight-year study). When interpreting results according to treatment received, it should be noted that by the time the analysis was completed, available therapies for CTEPH had failed to show a benefit in exercise capacity or survival in CTEPH patients [20-22]. Recently, two novelties have changed the CTEPH management scenario that might change prognosis in patients not undergoing PEA: riociguat, a recent treatment licensed for CTEPH and PAH, and secondly, the 
suggestion of pulmonary angioplasty as a supplementary interventional treatment option for inoperable CTEPH patients [19, 27].

\section{CONCLUSIONS}

The low rate of PEA reported in our national registry, and thus the important percentage of patients whom do not benefit from this potentially curative option, highlights that despite efforts made in CTEPH patient management such as increasing awareness and expertise in PEA at specialized centers, such efforts do not seem to translate into generalized benefits for patients when a decentralized model of decision making with respect to referral for PEA assessment is followed. Organizational models for CTEPH management should take these putative weaknesses into account and specifically ensure that all patients are timely referred for operability assessment as recommended by guidelines and expert committees [5, 19].

\section{ACKNOWLEDGEMENTS}

We express our gratitude to Bayer Schering Pharma for supporting this Registry with an unrestricted educational grant. We gratefully acknowledge all investigators who form part of the REHAP Registry. We also thank the Registry Coordinating Center, S \& H Medical Science Service, for their quality control, logistic and administrative support. We also thank Beatriz Viejo, $\mathrm{PhD}$ for her assistance in the writing of the manuscript and editorial support.

Members of the REHAP Group are the following: Artero ML, Hospital Universitario Virgen del Rocío, Sevilla; Barberá JA, Hospital Clinic i Provincial de Barcelona, 
Barcelona; Blanco I, Hospital Clinic i Provincial de Barcelona, Barcelona; Cadenas S, Hospital Clínico de Salamanca, Salamanca; Castillo MJ, Hospital Universitario Virgen del Rocío, Sevilla; Cortina J, Hospital Universitario Doce de Octubre, Madrid; Crémer D, Hospital Son Llatzer, Baleares; Domingo JA, Hospital Universitario Miguel Server, Zaragoza; Egurbide V, Hospital Universitario Cruces, Vizcaya; Elías T, Hospital Universitario Virgen del Rocío, Sevilla; Escribano P, Hospital Universitario Doce de Octubre, Madrid; Fortuna AM, Hospital de la Santa Creu i Sant Pau, Barcelona; García S, Hospital de León, León; García FJ, Hospital Universitario Virgen del Rocío, Sevilla; Jara L, Hospital Universitario Virgen del Rocío, Sevilla; Jiménez J, Hospital del SAS de Jerez de la Frontera, Cádiz; Juan-Samper G, Hospital General Universitario de Valencia, Valencia; Lara A, Hospital Universitario de Canarias, Santa Cruz de Tenerife; Lázaro M, Hospital Virgen de la Salud, Toledo; López-Ramón M, Hospital Universitario Miguel Servet, Zaragoza; Martínez F, Hospital Los Arcos, Murcia; Meseguer M, Hospital Vall d’Hebrón, Barcelona; Molina L, Hospital del Mar, Barcelona; Mombiela T, Hospital Universitario Gregorio Marañón, Madrid; Otero I, Hospital A Coruña, Coruña; Otero R, Hospital Universitario Virgen del Rocío, Sevilla; del Pozo R, Hospital Universitario Doce de Octubre, Madrid; Román A, Hospital Vall d’Hebrón, Barcelona; Segovia J, Hospital Universitario Puerta de Hierro, Madrid; Sala E, Hospital Universitario Son Dureta, Islas Baleares; Vicente M, Hospital Universitario Doce de Octubre, Madrid. 


\section{REFERENCES}

[1] McNeil K, Dunning J. Chronic thromboembolic pulmonary hypertension (CTEPH). Heart. 2007;93:1152-8.

[2] Hoeper MM, Mayer E, Simonneau G, Rubin LJ. Chronic thromboembolic pulmonary hypertension. Circulation. 2006;113:2011-20.

[3] Condliffe R, Kiely DG, Gibbs JS, Corris PA, Peacock AJ, Jenkins DP, et al. Improved outcomes in medically and surgically treated chronic thromboembolic pulmonary hypertension. Am J Respir Crit Care Med. 2008;177:1122-7.

[4] Escribano-Subias P, Blanco I, Lopez-Meseguer M, Lopez-Guarch CJ, Roman A, Morales P, et al. Survival in pulmonary hypertension in Spain: insights from the Spanish registry. Eur Respir J. 2012;40:596-603.

[5] Galie N, Hoeper MM, Humbert M, Torbicki A, Vachiery JL, Barbera JA, et al. Guidelines for the diagnosis and treatment of pulmonary hypertension: the Task Force for the Diagnosis and Treatment of Pulmonary Hypertension of the European Society of Cardiology (ESC) and the European Respiratory Society (ERS), endorsed by the International Society of Heart and Lung Transplantation (ISHLT). Eur Heart J. 2009;30:2493-537.

[6] Mayer E, Jenkins D, Lindner J, D'Armini A, Kloek J, Meyns B, et al. Surgical management and outcome of patients with chronic thromboembolic pulmonary hypertension: results from an international prospective registry. $\mathrm{J}$ Thorac Cardiovasc Surg. 2011;141:702-10.

[7] Corsico AG, D'Armini AM, Cerveri I, Klersy C, Ansaldo E, Niniano R, et al. Longterm outcome after pulmonary endarterectomy. Am J Respir Crit Care Med.

2008;178:419-24.

[8] Rahnavardi M, Yan TD, Cao C, Vallely MP, Bannon PG, Wilson MK. Pulmonary Thromboendarterectomy for Chronic Thromboembolic Pulmonary Hypertension : A Systematic Review. Annals of Thoracic and Cardiovascular Surgery. 2011;17:435-45.

[9] Ghofrani HA, D'Armini AM, Grimminger F, Hoeper MM, Jansa P, Kim NH, et al. Riociguat for the treatment of chronic thromboembolic pulmonary hypertension. N Engl J Med. 2013;369:319-29.

[10] ESC/ERS Guidelines for the diagnosis and treatment of pulmonary hypertension. Eur Heart J. 2015.

[11] Pepke-Zaba J, Delcroix M, Lang I, Mayer E, Jansa P, Ambroz D, et al. Chronic thromboembolic pulmonary hypertension (CTEPH): results from an international prospective registry. Circulation. 2011;124:1973-81.

[12] National Audit of Pulmonary Hypertension 2013. Report for the audit period 2012.

[13] Alonso-Gonzalez R, Lopez-Guarch CJ, Subirana-Domenech MT, Ruiz JM, Gonzalez IO, Cubero JS, et al. Pulmonary hypertension and congenital heart disease: An insight from the REHAP National Registry. Int J Cardiol. 2015;184:717-23. 
[14] Badesch DB, Champion HC, Sanchez MA, Hoeper MM, Loyd JE, Manes A, et al. Diagnosis and assessment of pulmonary arterial hypertension. J Am Coll Cardiol. 2009;54:S55-66.

[15] Freed DH, Thomson BM, Berman M, Tsui SS, Dunning J, Sheares KK, et al. Survival after pulmonary thromboendarterectomy: effect of residual pulmonary hypertension. J Thorac Cardiovasc Surg. 2011;141:383-7.

[16] Lang IM, Simonneau G, Pepke-Zaba JW, Mayer E, Ambroz D, Blanco I, et al. Factors associated with diagnosis and operability of chronic thromboembolic pulmonary hypertension. A case-control study. Thromb Haemost. 2013;110:83-91.

[17] Blazquez JA, Escribano P, Perez E, Lopez MJ, Gomez MA, Cortina JM. [Chronic thromboembolic pulmonary hypertension: surgical treatment with thromboendarterectomy]. Arch Bronconeumol. 2009;45:496-501.

[18] Coronel ML, Chamorro N, Blanco I, Amado V, Del Pozo R, Pomar JL, et al. Medical and Surgical Management for Chronic Thromboembolic Pulmonary Hypertension: A Single Center Experience. Arch Bronconeumol. 2014.

[19] Kim NH, Delcroix M, Jenkins DP, Channick R, Dartevelle P, Jansa P, et al. Chronic thromboembolic pulmonary hypertension. J Am Coll Cardiol. 2013;62:D92-9.

[20] Jais X, D'Armini AM, Jansa P, Torbicki A, Delcroix M, Ghofrani HA, et al. Bosentan for treatment of inoperable chronic thromboembolic pulmonary hypertension: BENEFiT (Bosentan Effects in iNopErable Forms of chronIc Thromboembolic pulmonary hypertension), a randomized, placebo-controlled trial. J Am Coll Cardiol. 2008;52:2127-34.

[21] Suntharalingam J, Treacy CM, Doughty NJ, Goldsmith K, Soon E, Toshner MR, et al. Long-term use of sildenafil in inoperable chronic thromboembolic pulmonary hypertension. Chest. 2008;134:229-36.

[22] Olschewski H, Simonneau G, Galie N, Higenbottam T, Naeije R, Rubin LJ, et al. Inhaled iloprost for severe pulmonary hypertension. N Engl J Med. 2002;347:322-9.

[23] Jensen KW, Kerr KM, Fedullo PF, Kim NH, Test VJ, Ben-Yehuda O, et al. Pulmonary hypertensive medical therapy in chronic thromboembolic pulmonary hypertension before pulmonary thromboendarterectomy. Circulation. 2009;120:124854.

[24] Raymond RJ, Hinderliter AL, Willis PW, Ralph D, Caldwell EJ, Williams W, et al. Echocardiographic predictors of adverse outcomes in primary pulmonary hypertension. J Am Coll Cardiol. 2002;39:1214-9.

[25] Benza RL, Miller DP, Gomberg-Maitland M, Frantz RP, Foreman AJ, Coffey CS, et al. Predicting survival in pulmonary arterial hypertension: insights from the Registry to Evaluate Early and Long-Term Pulmonary Arterial Hypertension Disease Management (REVEAL). Circulation. 2010;122:164-72. 
[26] Bonderman D, Skoro-Sajer N, Jakowitsch J, Adlbrecht C, Dunkler D, Taghavi S, et al. Predictors of outcome in chronic thromboembolic pulmonary hypertension.

Circulation. 2007;115:2153-8.

[27] Dimopoulos K, Kempny A, Alonso-Gonzalez R, Wort SJ. Percutaneous transluminal pulmonary angioplasty for the treatment of chronic thromboembolic pulmonary hypertension: Challenges and future directions. Int J Cardiol. 2015;187:4013. 
Table 1. Patients' characteristics at diagnosis.

Variables

Demographic and anthropometric

Gender, n (\%) males

Age, years, median $(\mathrm{Q} 1 ; \mathrm{Q} 3)$

BMI, $\mathrm{kg} / \mathrm{m}^{2}$, mean $(\mathrm{SD})^{\mathrm{a}}$

\section{Clinical}

WHO FC I / II, n (\%)

WHO FC III, n (\%)

WHO FC IV, n (\%)

6MWD, m, median $(\mathrm{Q} 1 ; \mathrm{Q} 3)^{\mathrm{b}}$

\section{Hemodynamic}

Pericardial effusion, $\mathrm{n}(\%)^{\mathrm{c}}$

\section{CTEPH}

PEA

non-PEA

$\mathrm{P}$

$$
(\mathrm{n}=391) \quad(\mathrm{n}=122) \quad(\mathrm{n}=269)
$$

$\begin{array}{cccc}164(42.0) & 68(56.0) & 96(36.0) & <\mathbf{0 . 0 0 1} \\ 63.7(48.0 ; 73.3) & 50(41 ; 65) & 69(55 ; 75) & <\mathbf{0 . 0 0 1} \\ 28.3(5.3) & 27.5(5.1) & 28.6(5.4) & 0.063\end{array}$

$\begin{array}{cccc}114(29.0) & 34(28.0) & 80(30.0) & 0.816 \\ 249(64.0) & 83(68.0) & 166(62.0) & 0.257 \\ 28(7.0) & 5(4.1) & 23(8.6) & 0.140 \\ & & & \\ 440(243.5 ; 432.0) & 400(290 ; 475) & 320(210 ; 407) & \mathbf{0 . 0 0 1}\end{array}$

$35(9.9 \%)$

$14(13.0)$

$21(8.5)$

0.181 
mPAP, mmHg, mean $(\mathrm{SD})^{\mathrm{d}}$

PVR, Wood units, median $(\mathrm{Q} 1 ; \mathrm{Q} 3)^{\mathrm{e}}$

Cardiac output, mean (SD) ${ }^{\mathrm{f}}$

$$
45.6(12.4)
$$

8.5

$(5.6 ; 12.4)$

$4.2(1.2)$

$$
48.0(12.6)
$$

8.6

$(6.2-13.1)$

$4.2(1.1)$
44.5 (12.2)

8.3

0.011

0.461

(5.4-12.4)

BMI: Body mass index; WHO FC: World Health Organization Functional Class; 6MWD: 6-min walking distance; mPAP: Mean pulmonary arterial pressure; PVR: Pulmonary vascular resistance.

Missing values: ${ }^{\mathrm{a}} 18$ in PEA, 3 in non-PEA; 32 in PEA, 39 in non-PEA; ${ }^{\mathrm{c}} 15$ in PEA, 21 in non-PEA; 5 in PEA, 30 in non-PEA; 3 in PEA, 14 in non-PEA; ${ }^{2}$ in PEA, 13 in non-PEA 
Table 2. Treatment administered to patients not undergoing PEA $(n=269)$.

\begin{tabular}{lc}
\hline Medical treatment & $\mathbf{n}(\%)$ \\
\hline No treatment & $49(18.2)$ \\
PDE-5i & $84(31.2)$ \\
ERA & $103(38.3)$ \\
Inhaled prostacyclin analogues & $1(0.4)$ \\
Subcutaneous prostacyclin analogues & $0(0)$ \\
Intravenous prostacyclin analogues & $3(1.1)$ \\
Oral combined therapy & $22(8.2)$ \\
Inhaled prostacyclin + oral therapy & $2(0.7)$ \\
Subcutaneous prostacyclin analogues + & $2(0.7)$ \\
oral therapy & \\
Intravenous prostacyclin analogues + oral \\
therapy
\end{tabular}

PDE-5i: Phosphodiesterase 5 inhibitor; ERA: Endothelin receptor antagonist. 
Table 3. Patients' characteristics associated with mortality in univariate analysis (hazard ratio).

\begin{tabular}{|c|c|c|c|c|c|c|}
\hline \multirow[b]{2}{*}{ Variables } & \multicolumn{2}{|c|}{ Overall population $(\mathrm{n}=391)$} & \multicolumn{2}{|c|}{ PEA $(n=122)$} & \multicolumn{2}{|c|}{ Non-PEA $(n=269)$} \\
\hline & $\mathrm{HR}(95 \% \mathrm{CI})$ & $\begin{array}{c}\mathrm{P}- \\
\text { value* }\end{array}$ & $\mathrm{HR}(95 \% \mathrm{CI})$ & $\begin{array}{c}\text { P- } \\
\text { value* }\end{array}$ & $\operatorname{HR}(95 \% \mathrm{CI})$ & $\begin{array}{c}\text { P- } \\
\text { value* }\end{array}$ \\
\hline Demographic and anthropometric & & & & & & \\
\hline Male gender & $0.88(0.56 ; 1.38)$ & 0.589 & $0.55(0.18 ; 1.67)$ & 0.295 & $1.18(0.72 ; 1.92)$ & 0.515 \\
\hline Age, HR per 10 years & $1.30(1.10 ; 1.53)$ & 0.002 & $1.06(0.72 ; 1.55)$ & 0.777 & $1.25(1.02 ; 1.53)$ & $\mathbf{0 . 0 3 0}$ \\
\hline $\mathrm{BMI}^{\mathrm{a}} \geq 30 \mathrm{~kg} / \mathrm{m}^{2}$ & $0.48(0.27 ; 0.85)$ & 0.013 & $0.50(0.13 ; 1.86)$ & 0.299 & $0.48(0.25 ; 0.92)$ & 0.026 \\
\hline Clinical parameters & & & & & & \\
\hline WHO FC I and II & $0.33(0.17 ; 0.61)$ & 0.001 & $0.03(0.00 ; 3.73)$ & 0.153 & $0.39(0.20 ; 0.74)$ & 0.004 \\
\hline WHO FC III & $1.48(0.92 ; 2.37)$ & 0.108 & $6.40(0.84 ; 49.0)$ & 0.074 & $1.27(0.77 ; 2.09)$ & 0.351 \\
\hline WHO FC IV & $3.13(1.73 ; 5.67)$ & $<0.001$ & $2.03(0.26 ; 15.7)$ & 0.495 & $3.24(1.73 ; 6.04)$ & $<0.001$ \\
\hline Syncope & $0.53(0.25 ; 1.16)$ & 0.113 & $1.15(0.32 ; 4.12)$ & 0.833 & $0.39(0.14 ; 1.08)$ & 0.069 \\
\hline $6 \mathrm{MWD}^{\mathrm{b}}, \mathrm{HR}$ per $20 \mathrm{~m}$ & $0.93(0.90 ; 0.96)$ & $<0.001$ & $0.96(0.88 ; 1.04)$ & 0.310 & $0.93(0.90 ; 0.97)$ & $<0.001$ \\
\hline $\begin{array}{l}\text { Hemodynamic parameters } \\
\text { Echocardiography }\end{array}$ & & & & & & \\
\hline
\end{tabular}




\begin{tabular}{|c|c|c|c|c|c|c|}
\hline $\mathrm{PSP}^{\mathrm{c}}, \mathrm{HR}$ per $1 \mathrm{mmHg}$ & $1.01(1.00 ; 1.02)$ & 0.211 & $1.01(0.99 ; 1.04)$ & 0.262 & $1.01(0.99 ; 1.02)$ & 0.296 \\
\hline Pericardial effusion ${ }^{\mathrm{d}}$ & $2.57(1.44 ; 4.61)$ & 0.001 & $3.71(1.20 ; 11.5)$ & 0.023 & $3.06(1.50 ; 6.26)$ & 0.002 \\
\hline \multicolumn{7}{|l|}{ Right heart catheterisation } \\
\hline $\mathrm{mPAP}^{\mathrm{e}}, \mathrm{HR}$ per $5 \mathrm{~mm} \mathrm{Hg}$ & $1.05(0.97 ; 1.14)$ & 0.200 & $1.12(0.93 ; 1.36)$ & 0.231 & $1.08(0.98 ; 1.18)$ & 0.102 \\
\hline RAP, HR per $5 \mathrm{~mm} \mathrm{Hg}$ & $1.39(1.15 ; 1.67)$ & 0.001 & $1.61(1.05 ; 2.47)$ & 0.028 & $1.37(1.11 ; 1.69)$ & 0.004 \\
\hline Cardiac output ${ }^{\mathrm{f}}, \mathrm{HR}$ per $0.5 \mathrm{~L} \cdot \mathrm{min}^{-1}$ & $0.80(0.71 ; 0.89)$ & $<0.001$ & $0.73(0.55 ; 0.97)$ & 0.029 & $0.82(0.73 ; 0.92)$ & 0.001 \\
\hline PVR $^{\mathrm{g}}$, HR per 3 Wood units & $1.17(1.07 ; 1.29)$ & 0.001 & $1.41(1.04 ; 1.90)$ & 0.025 & $1.15(1.04 ; 1.26)$ & 0.007 \\
\hline \multicolumn{7}{|l|}{ Respiratory parameters } \\
\hline $\mathrm{FVC}<60 \%^{\mathrm{h}}$ & $1.72(0.85 ; 3.47)$ & 0.132 & $4.90(1.04 ; 23.2)$ & 0.045 & $1.31(0.59 ; 2.91)$ & 0.499 \\
\hline $\mathrm{FEV}_{1}<60 \%{ }^{\mathrm{i}}$ & $1.98(1.10 ; 3.59)$ & 0.024 & $3.34(0.41 ; 27.3)$ & 0.260 & $1.66(0.89 ; 3.09)$ & 0.114 \\
\hline Proximal lesions $^{\dagger j}$ & $1.17(0.73 ; 1.89)$ & 0.505 & $0.46(0.13 ; 1.62)$ & 0.224 & $2.62(1.54 ; 4.43)$ & $<0.001$ \\
\hline Undergoing PEA & $0.42(0.24 ; 0.75)$ & 0.003 & - & - & - & - \\
\hline Time from diagnosis to PEA / MT & $1.00(1.00 ; 1.00)$ & 0.068 & $1.00(1.00 ; 1.00)$ & 0.456 & $1.00(1.00 ; 1.00)$ & 0.295 \\
\hline Pre-treatment before surgery & - & - & $1.25(0.44 ; 3.60)$ & 0.675 & - & - \\
\hline
\end{tabular}


$*$ P-values $<0.2$ at the Univariate Cox's proportional-hazard regression model are indicated in bold.

${ }^{\dagger}$ Lesions were assessed by computed tomographic angiography. Those affecting main and lobar arteries were considered proximal lesions.

BMI: Body mass index; WHO FC: World Health Organization Functional Class; 6MWD: 6-min walking distance; PSP: Pulmonary systolic pressure; mPAP: Mean

pulmonary arterial pressure; RAP: Right atrial pressure; PVR: Pulmonary vascular resistance; FVC: Forced vital capacity; FEV ${ }_{1}$ Forced expiratory volume in 1 second; PEA:

Pulmonary endarterectomy; MT: medical treatment.

Missing values: ${ }^{\mathrm{a}} 18$ in PEA, 3 in non-PEA; ${ }^{\mathrm{b}} 32$ in PEA, 39 in non-PEA; ${ }^{\mathrm{c}} 16$ in PEA, 26 in non-PEA; ${ }^{\mathrm{d}} 15$ in PEA, 21 in non-PEA; 5 in PEA, 30 in non-PEA; 2 in PEA, 13 in non-PEA; ${ }^{\mathrm{g}} 3$ in PEA, 14 in non-PEA; ${ }^{\mathrm{h}} 23$ in PEA, 51 in non-PEA; 22 in PEA, 45 in non-PEA; ${ }^{\mathrm{j}} 30$ in PEA, 47 in non-PEA. 


\section{FIGURE LEGENDS}

Figure 1. Operability of patients in the REHAP registry.

*Patients assessed in these centers include those belonging to the health administrative area and those being referred from local $\mathrm{PH}$-specialized centers / general hospitals. 
Figure 2. Kaplan-Meier estimates of cumulative survival from diagnosis (years) in PEA and non-PEA patients.

$\mathrm{P}=0.003$

\begin{tabular}{|l|c|c|c|c|c|}
\hline Patients at risk & $\mathbf{0 - 1}$ year & $\mathbf{1 - 2}$ years & $\mathbf{2 - 3}$ years & $\mathbf{3 - 4}$ years & $\mathbf{4 - 5}$ years \\
\hline PEA & 114 & 89 & 65 & 50 & 39 \\
\hline Non-PEA & 260 & 212 & 160 & 118 & 84 \\
\hline
\end{tabular}


Figure 3. Characteristics associated with mortality in multivariate analysis in the overall population and in PEA and non-PEA patients (hazard ratio).

6MWD: 6-min walking distance; PEA: pulmonary endarterectomy. HR analyzed are indicated in table 3. 
Figure 4. Hemodynamic and clinical improvement achieved at the follow up visit for patients who undergone PEA and those who did not (Delta values).

SD: standard deviation; WHO FC: WHO functional class; 6MWD: 6-min walking distance; mPAP: Mean pulmonary arterial pressure.

Data were missing for 25 patients due to death ( $\mathrm{n}=6$ in the PEA group, $\mathrm{n}=19$ in the non-PEA group), due to undergoing PEA or starting medical treatment after December, 31 2013, with visits taking place after the pre-determined follow-up period ( $\mathrm{n}=13$ and $\mathrm{n}=3$, respectively), or due to lack of a follow-up visit during the follow-up period ( $\mathrm{n}=4$ and $\mathrm{n}=10$, respectively). 


\section{SUPPLEMENTAL MATERIAL}

Table 1. Differential characteristics between patients assessed at expert centers* and in other centers.

\begin{tabular}{|l|cc|c|}
\hline Variables & $\begin{array}{c}\text { Expert centers } \\
(\mathrm{N}=240)\end{array}$ & $\begin{array}{c}\text { Other centers } \\
(\mathrm{N}=151)\end{array}$ & P value \\
\hline Demographic and anthropometric & $102(42)$ & $62(42)$ & 1.000 \\
Gender, n (\%) males & $57.1(44.6 ; 69.3)$ & $70.4(58.3 ; 76.4)$ & $<\mathbf{0 . 0 0 1}$ \\
Age, years, median (Q1;Q3) & $28(5.0)$ & $28.6(5.8)$ & 0.352 \\
BMI, kg/m ${ }^{2}$, mean (SD) & $160(66)$ & $89(60)$ & 0.234 \\
\hline Clinical parameters & $14(5.8)$ & $14(9.4)$ & 0.225 \\
WHO FC III, n (\%) & $41(17.0)$ & $13(8.8)$ & $\mathbf{0 . 0 2 4}$ \\
WHO FC IV, n (\%) & $368(258 ; 450)$ & $305(207 ; 385)$ & $\mathbf{0 . 0 0 2}$ \\
Syncope, n (\%) & $96(49)$ & $27(23)$ & $<\mathbf{0 . 0 0 1}$ \\
6MWD, m, median (Q1;Q3) & $30(14.0)$ & $5(3.7)$ & $\mathbf{0 . 0 0 2}$ \\
\hline Hemodynamic parameters & $47.1(12.3)$ & $43.1(12.2)$ & $\mathbf{0 . 0 0 2}$ \\
Pericardial effusion, n (\%) & $9.3(6.0 ; 14.0)$ & $7.2(5.3 ; 10.5)$ & $\mathbf{0 . 0 1 3}$ \\
mPAP, mmHg, mean (SD) & $4.1(1.1)$ & & $\mathbf{0 . 0 2 7}$ \\
PVR, Wood units, median (Q1;Q3) & & & \\
Cardiac output, mean (SD) & & & \\
\hline Proximal lesions & & & \\
\hline
\end{tabular}

*CTEPH expert centers are Hospital 12 de Octubre (Madrid) and Hospital Clínic (Barcelona). Patients assessed in these centers include those belonging to the health administrative area and those being referred from local $\mathrm{PH}$-specialized centers.

BMI: Body mass index; WHO FC: World Health Organization Functional Class; 6MWD: 6-min walking distance; PSP: Pulmonary systolic pressure; mPAP: Mean pulmonary arterial pressure; RAP: Right atrial pressure; PVR: Pulmonary vascular resistance.

Missing values for each variable are indicated in Table 3 (manuscript). 
Table 2. Patients' characteristics associated with operability in univariate analysis (hazard ratio).

\begin{tabular}{|c|c|c|}
\hline Variables & $\begin{array}{l}\text { HR }(95 \% \mathrm{CI}) \text { for } \\
\text { operability }\end{array}$ & $P$ value \\
\hline \multicolumn{3}{|l|}{ Demographic and anthropometric } \\
\hline Male gender & $2.27(1.47 ; 3.51)$ & $<0.001$ \\
\hline Age, HR per 10 years & $0.58(0.50 ; 0.68)$ & $<0.001$ \\
\hline $\mathrm{BMI} \geq 30 \mathrm{~kg} / \mathrm{m}^{2}$ & $0.81(0.49 ; 1.33)$ & 0.399 \\
\hline \multicolumn{3}{|l|}{ Clinical parameters } \\
\hline WHO FC I and II, & $0.91(0.57 ; 1.47)$ & 0.706 \\
\hline WHO FC III & $1.32(0.84 ; 2.08)$ & 0.229 \\
\hline WHO FC IV & $0.46(0.17 ; 1.23)$ & 0.122 \\
\hline Syncope & $1.78(0.99 ; 3.20)$ & 0.056 \\
\hline $6 \mathrm{MWD}, \mathrm{HR}$ per $20 \mathrm{~m}$ & $1.08(1.04 ; 1.13)$ & $<0.001$ \\
\hline \multicolumn{3}{|l|}{ Hemodynamic parameters } \\
\hline PSP, HR per $1 \mathrm{~mm} \mathrm{Hg}$ & $1.00(0.99 ; 1.01)$ & 0.956 \\
\hline $\begin{array}{l}\text { Pericardial effusion } \\
\text { Right heart catheterisation }\end{array}$ & $1.63(0.79 ; 3.34)$ & 0.184 \\
\hline mPAP, HR per $5 \mathrm{mmHg}$ & $1.12(1.03 ; 1.22)$ & 0.011 \\
\hline RAP, HR per $5 \mathrm{mmHg}$ & $1.04(0.85 ; 1.28)$ & 0.686 \\
\hline Cardiac output, HR per $0.5 \mathrm{~L} \cdot \mathrm{min}^{-1}$ & $1.03(0.93 ; 1.13)$ & 0.578 \\
\hline PVR, HR per 3 Wood units & $1.02(0.91 ; 1.14)$ & 0.765 \\
\hline Respiratory parameters & & \\
\hline
\end{tabular}




\begin{tabular}{|l|cc|} 
FVC $<60 \%$ & $0.50(0.18 ; 1.36)$ & $\mathbf{0 . 1 7 6}$ \\
FEV $_{1}<60 \%$ & $0.23(0.09 ; 0.61)$ & $\mathbf{0 . 0 0 3}$ \\
\hline Proximal lesions $^{\dagger}$ & $8.60(4.93 ; 15.00)$ & $<\mathbf{0 . 0 0 1}$ \\
\hline
\end{tabular}

*P-values $<0.2$ at the Univariate Cox's proportional-hazard regression model are indicated in bold.

BMI: Body mass index; WHO FC: World Health Organization Functional Class; 6MWD: 6-min walking distance; PSP: Pulmonary systolic pressure; mPAP: Mean pulmonary arterial pressure; RAP: Right atrial pressure; PVR: Pulmonary vascular resistance; FVC: Forced vital capacity; $F_{E V}$ : Forced expiratory volume in 1 second.

Missing values for each variable are indicated in Table 3 (manuscript). 
Figure 1. Multivariate analysis of characteristics associated with operability (hazard ratio).

6MWD: 6-min walking distance. HR analyzed are indicated in Table 2 at this Supplementary Appendix. 